\title{
Diabetes-Cancer Risk: Hyperglycemia on DNA Damage and Repair
}

\author{
Amy Zhong ${ }^{1}$, Yumay Chen ${ }^{1,2 *}$ and Phang Lang Chen ${ }^{3 *}$ \\ ${ }^{1}$ Diabetic Center, University of California, USA \\ ${ }^{2}$ Department of Medicine/Endocrinology, University of California, USA \\ ${ }^{3}$ Department of Biological Chemistry, University of California, USA
}

*Corresponding author: Yumay Chen, Department of Medicine/Endocrinology, University of California, Irvine, 1130 Gross Hall, Zot Code: 4086. Irvine, CA 92697, USA.

Phang Lang Chen, Department of Biological Chemistry, University of California, Irvine, D252 Med Sci 1, Zot code: 1700, Irvine, CA 92697, USA

To Cite This Article: Yumay Chen. Diabetes-Cancer Risk: Hyperglycemia on DNA Damage and Repair. Am J Biomed Sci \& Res. 2019 - 5(4). AJBSR. MS.ID.000938. DOI: 10.34297/AJBSR.2019.05.000939.

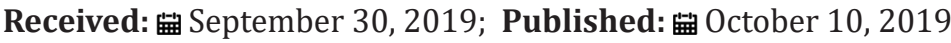

\begin{abstract}
Increased risk of certain cancers has been reported in populations with diabetes mellitus. Ample research supports the notion that diabetic conditions generate excessive amounts of reactive oxygen species, thus resulting in oxidative DNA damage. Recently, several discoveries have shown that cells grown in high glucose conditions also have defective DNA damage response and repair pathways. When considered together, the dual concerns of elevated oxidative DNA damage and aberrant DNA damage response and repair underscore the importance of maintaining tight glycemic control in patients with diabetes mellitus and cancer.

Keywords: Cancer; Diabetes; DNA damage response pathway; NEK1; ATM; ATR; Hyperglycemia

Abbreviations: ATM: Ataxia Telangiectasia Mutated; ATR: ATM and Rad3-related kinase; NEK1: Nima A Related Protein Kinase 1; $\gamma$ H2AXphosphorylation of H2AX at Ser 139; IR: Ionizing Radiation; CIN: Chromosomal Instability; GLUT2: Glucose transporter 2; CHK1: Cell-Cycle Checkpoint Kinase 1; VDAC1: Voltage Dependent Anion Channel 1; MPTP: Mitochondrial Permeability Transition Pore; ANT: Adenine Nucleotide Translocator; CyoD: Cyclophilin D
\end{abstract}

\section{Introduction}

Faithful replication and transmission of genetic material to daughter cells is fundamental to all life forms. To maintain genome stability, cells must safeguard the DNA passed onto their daughter cells. Thus, the ability to recognize and repair DNA damage generated by endogenous or exogenous agents is essential in preventing errors from being passed onto daughter cells during cell division. This error detection mechanism, known as the DNA damage-repair signaling pathway, functions by sensing the DNA damage and generating signals to arrest cell cycle progression, thereby allowing for the DNA damage lesion to be repaired in a timely manner.

Through modulating a group of protein kinases and sensors, the DNA damage-repair signaling network activates a series of checkpoints that temporarily halts the progression of the cell cycle [1]. This prevents the cell from replicating its DNA or delays mitosis until DNA can be assessed and repaired. The sensor proteins in the DNA damage-repair signaling network include ATM (ataxia telangiectasia mutated), ATR (ATM and Rad3-related kinase) and NEK1 (Nima A related protein kinase) kinase [2-4]. ATM, ATR, and NEK1 work independently and activate similar intersecting downstream targets which are important for signaling and repairing DNA double-strand breaks. Among these three kinases, ATR is thought to be the most crucial upstream phosphatidylinositol 3-kinase (PI3K) for signaling and repairing UV radiation-induced and nucleoside analog-induced DNA damage, both of which cause stalled replication forks $[5,6]$. NEK1 appears to oversee DNA damage responses in some ways similar to ATM and ATR, but it is unique and independent of both canonical kinases. Like ATMdeficient and ATR-deficient cells, NEK1-deficient (through mutation or silencing by RNAi) cells are much more sensitive to the effects of ionizing radiation (IR)-induced DNA damage than the wild type cells [3,7]. NEK1-deficient cells do not repair damaged DNA as efficiently as control cells and have defective checkpoints $[2,3,8,9]$. 
Like ATM and ATR mutant mice, mice heterozygous for NEK1 also exhibit increased cancer incidence $[8,10]$. These NEK1 associated cancers have chromosomal instability (CIN) phenotypes, which are consistent with the notion that NEK1 mutant cells are defective in checkpoint regulation and result in elevated chromosome breaks, defective chromatid pairing, and mitotic mis segregation.

Just as DNA damage is a leading cause for cancer, DNA damage has also been suggested as a major contributing factor for the complications seen in diabetes [11]. Increased oxidative stress under hyperglycemic conditions has been well documented [12]. When untransformed primary renal epithelial cells were grown under hyperglycemic conditions, mutation rates increased. In addition, increased $\gamma$ H2AX signals, a quantitative marker of DNA double strand breaks, can be detected in cells grown in high glucose conditions [13]. This implies that cells grown in high glucose conditions, like those of untreated diabetic patients, could have more DNA double-strand breaks than those maintained in normal glucose conditions. In animal models, mice had more DNA breaks in pancreas when fed with high sugar/high fat diets than those fed with a standard chow diet [14]. Glucose enters the cell through GLUT2 glucose transporter [15], so high expression levels of GLUT2 in the pancreas can begin to explain why DNA breaks are primary found in pancreas, as opposed to in other organs.

In the DNA damage response pathway, both ATM and ATR are believed to be crucial proximal signaling molecules in all forms of DNA damage sensing and repair [1]. Unlike ATM, ATR and NEK1 also function as stress response proteins [13]. NEK1, as a general stress response protein, responds to hyperosmolar/low glucose or high glucose conditions. ATR, on the other hand, is a more selective stress response protein which could only be activated under high glucose conditions, not hyperosmolar/low glucose conditions. Although ATR and NEK1 protein kinases are both activated under high glucose conditions, the downstream checkpoint response protein, CHK1, is not activated, which results in the failure to properly repair damaged DNA [13]. This observation suggests there is a defect somewhere between ATR and CHK1 in the DDR pathway under hyperglycemic conditions which results in mutation accumulation. Identifying the critical cellular and molecular factors affected by the high glucose condition will begin to provide molecular insights that can pave ways to novel therapeutics.

Unlike ATM and ATR, NEK1 is also involved directly in the apoptosis pathway, through the regulation of the mitochondrial outer membrane protein VDAC1 [16,17]. Apoptosis is an intrinsic cell death mechanism, which removes damaged cells before passing genetic mutations onto a subsequent generation of cells, often without triggering an inflammatory response in neighboring cells [18]. During oxidative injury and DNA damage induced cell death, the mitochondrial membrane potential (MPM) is collapsed through the opening of the mitochondrial permeability transition pore (MPTP).
With the loss of MPM, cytochrome $\mathrm{C}$ is released from mitochondria into the cytosol and activates the caspase cascade to initiate apoptosis. MPTP composes of VDAC1, the inner mitochondrial membrane protein ANT (adenine nucleotide translocator), and the inner mitochondrial membrane protein cyclophilin D (cypD) [19]. Through interaction and phosphorylation of VDAC1, NEK1 regulates the release of cytochrome $\mathrm{C}$ from mitochondria in the initiation step of apoptosis. In the basal state of a cell, NEK1 interacts with and phosphorylates VDAC1, and seems to keep the mitochondrial transition pore intact [16]. Upon being exposed to genotoxic agents, NEK1 dissociates with VDAC1, and VDAC1 become dephosphorylated, thus inducing the apoptosis pathway. Therefore, the expression levels of NEK1 determine the genotoxic sensitivity in given cells. Higher doses of DNA damaging agents are needed to kill cells with high expression levels of NEK1 [20]. Glucose induced up-regulation of NEK1 may not only contribute directly to DNA damage response, but also to cell survival. In hyperglycemic conditions, cells are more resistant to genotoxic agents [13]. This observation is very critical, since it implies that hyperglycemia may make cells more resistant to chemotherapy and radiation therapy. It also suggests that cells might easily become polyploids and transform into neoplastic cells when exposed to low doses of genotoxic agents in high glucose conditions.

Animal studies suggest that high glucose conditions can trigger a metabolic imbalance due to the downregulation of phosphofructokinase, which then leads to genomic mutations [14]. The published results reveal the mechanism behind how high glucose leads to genomic instability: mitochondrial mediated apoptosis/autophagy, DNA damage response/repair signaling, and a perturbed sugar metabolism. DNA damage agents have been used to treat cancers for decades. Given this new understanding of how hyperglycemic conditions influence the efficacy of DNA damaging agents, tighter glycemic control may be important for patients undergoing chemotherapy and radiation therapy with or without surgery for their cancer treatment.

\section{Conclusion}

Cancer and diabetes mellitus are two common diseases that frequently overlap in patients and account for high mortality worldwide. Population studies have established a strong link between cancer and diabetes. DNA damage has been suggested as a major contributing factor for both cancer and diabetes. Thus, as means to prevent DNA damage and maintain genomic stability and integrity, multiple surveillance mechanisms, including DNA repair, DNA damage checkpoints, and apoptosis, have evolved. Understanding how these surveillance mechanisms function and how glucose influences them will provide insight into how to design better therapeutic strategies for cancer and diabetes.

\section{Competing Financial Interests}

None 


\section{Author's Contributions}

AZ, YC and PLC drafted, edited and approved the final manuscript.

\section{Reference}

1. Shiloh Y (2006) The ATM-mediated DNA-damage response: taking shape. Trends Biochem Sci 31(7): 402-410.

2. Chen Y, Chen CF, Riley DJ, Chen PL (2011) Nek1 kinase functions in DNA damage response and checkpoint control through a pathway independent of ATM and ATR. Cell Cycle 10(4): 655-663.

3. Chen, Y, Chen PL, Chen CF, Jiang X, Riley DJ (2008) Never-in-mitosis related kinase 1 functions in DNA damage response and checkpoint control. Cell Cycle 7(20): 3194-3201.

4. McGowan CH, P Russell (2004) The DNA damage response: sensing and signaling. Curr Opin Cell Biol 16(6): 629-633.

5. Byun TS, Pacek M, Yee MC, Walter JC, Cimprich KA (2005) Functional uncoupling of MCM helicase and DNA polymerase activities activates the ATR-dependent checkpoint. Genes Dev 19(9): 1040-1052.

6. Unsal Kacmaz, K, Makhov AM, Griffith JD, Sancar A (2002) Preferential binding of ATR protein to UV-damaged DNA. Proc Natl Acad Sci U S A 99(10): 6673-6678.

7. Pelegrini AL, Moura DJ, Brenner BL, Ledur PF, Maques GP, et al. (2010) Nek1 silencing slows down DNA repair and blocks DNA damage-induced cell cycle arrest. Mutagenesis 25(5): 447-454.

8. Chen Y, Chi Fen Chen, Huai Chin Chiang, Michelle Pena, Rosaria Polci, et al (2011) Mutation of NIMA-related kinase 1 (NEK1) leads to chromosome instability. Mol Cancer 10(1): 1-5.

9. Polci R, Peng A, Chen PL, Riley DJ, Chen Y, et al. (2004) NIMA-related protein kinase 1 is involved early in the ionizing radiation-induced DNA damage response. Cancer Res 64(24): 8800-8803.
10. Xu Y, Ashley T, Brainerd EE, Bronson RT, Meyn MS, et al. (1996) Targeted disruption of ATM leads to growth retardation, chromosomal fragmentation during meiosis, immune defects, and thymic lymphoma. Genes Dev 10(19): 2411-2422.

11. Vigneri P, Frasca F, Sciacca L, Pandini G, Vigneri R (2009) Diabetes and cancer. Endocr Relat Cancer 16(4): 1103-1123.

12. Newsholme P, Cruzat VF, Keane KN, Carlessi R, de Bittencourt PI Jr (2016) Molecular mechanisms of ROS production and oxidative stress in diabetes. Biochem J 473(24): 4527-4550.

13. Zhong A, Melissa Chang, Theresa Yu, Raymond Gau, Daniel J Riley, et al. (2018) Aberrant DNA damage response and DNA repair pathway in high glucose conditions. J Can Res Updates 7(3): 64-74.

14. Hu CM, Tien SC, Hsieh PK, Jeng YM, Chang MC, et al. (2019) High Glucose Triggers Nucleotide Imbalance through 0-GlcNAcylation of Key Enzymes and Induces KRAS Mutation in Pancreatic Cells. Cell Metab 29(6): 13341349.

15. Szkudelski $\mathrm{T}$ (2001) The mechanism of alloxan and streptozotocin action in B cells of the rat pancreas. Physiol Res 50(6): 537-546.

16. Chen Y, WJ Craigen, DJ Riley (2009) Nek1 regulates cell death and mitochondrial membrane permeability through phosphorylation of VDAC1. Cell Cycle 8(2): 257-267.

17. Chen Y, Gaczynska M, Osmulski P, Polci R, Riley DJ (2010) Phosphorylation by Nek1 regulates opening and closing of voltage dependent anion channel 1. Biochem Biophys Res Commun 394(3): 798-803.

18. Kroemer G, L Galluzzi, C Brenner (2007) Mitochondrial membrane permeabilization in cell death. Physiol Rev 87(1): 99-163.

19. Tsujimoto Y, S Shimizu (2002) The voltage-dependent anion channel: an essential player in apoptosis. Biochimie 84(2-3): 187-193.

20. Chen Y, Chi Fen Chen, Rosaria Polci, Randy Wei, Daniel J Riley (2014) Increased Nek1 expression in renal cell carcinoma cells is associated with decreased sensitivity to DNA-damaging treatment. Oncotarget 5(12): 4283-4294. 